3. Frequent careful cleaning of the tube is of the greatest importance.

4. The few annoying sequelæ attending the use of the drainage tube can be avoided or easily cured.

5. It is safest to use the drainage tube too often, rather than too rarely, as we would in case of doubt use any other precautionary measure.

\section{RECOVERY FROM SYMPATHETIC OPH- THALMIA INDUCED BY A SARCOMA OF THE CHOROID.}

Read in the Section of Ophthalmology at the Fortieth Annual Meeting of the American Medical Association, June, 1889 .

BY F. C. HOTZ, M.D.,

\author{
of chicago.
}

Mr. M., aged 56 years, a robust man of regular habits, noticed in August, I887, a gradual failing of the sight of his right eye; and by the end of September the sight was entirely extinguished. Up to that time he had no pain or unpleasant feeling in or about his eye; but during October he had frequent paroxysms of violent pain in the blind eye and extending over the right side of the head.

October 29 , after a very severe attack of this headache, Mr. M. called for a consultation, and the examination of the eye showed the following conditions :

"Slight œdema of the upper lid; fulness of conjunctival and episcleral veins; hazy and lusterless cornea; shallow anterior chamber; pupil immobile and dilated ad maximum; lens transparent, but vitreous absolutely impenetrable for light; T. $+3:$ no perception. L. E. V. $=\frac{2}{2} \frac{0}{0}$ with + 2 D.; normal fundus."

As the sight had been slowly and painlessly extinguished before any of those violent symptoms of glaucoma appeared, it seemed most likely the glaucomatous state was caused by an intraocular tumor. The removal of the eye, therefore, was urgently advised, but as usual, the patient was not prepared to accept this advice at once. However, after he had gone through a few more and exceedingly severe attacks of hemicrania, he became very anxious to be relieved of the source of his suffering; and November 5 the eye was enucleated; the instruments were disinfected with carbolic acid ( 5 per cent.); the eye and lid was washed with sublimate solution ( $1: 5000)$; the wound was closed with aseptic silk and dusted over with iodoform.

Neither on the day of the operation nor on the two following days when I visited the patient at his house, anything unusual could be noticed in the external appearance of the left eye; nor was there any particular occasion for using the ophthalmoscope on those days, as the patient did not observe any disturbance of the sight, and de- scribed the feeling in his left eye as perfectly natural. As the healing progressed very satisfactorily the patient was instructed to call at the office in two or three days. He did not come until November I 2 because the right eye was doing well; but since two days, he said, the left eye had been getting red, sensitive to light, and the sight seemed to grow a little dim. This was found to be true, V. being only $\frac{2}{3} \frac{0}{0}$; slight pericorneal injection; clear cornea and anterior chamber; pupil of medium size; numerous posterior synechiæ which however were all easily broken by a few instillations of duboisine. When the pupil was fully dilated its field presented a very peculiar appearance under focal illumination. In the center there was a clear area exactly corresponding in size and form to the form of the pupil before duboisine had been used. The outline of this clear space was marked by a circle of very fine brownish dots (pigment from the synechiæ), and beyond this circle the anterior surface of the lens was covered with a film made up of innumerable thin and short fibres of a light brown color, irregularly curved and bent and promiscuously mixed together into a loosely woven network. Plainly visible though this film was by focal light, it offered surprisingly little obstruction to the ophthalmoscopic examination by which no disturbance in the vitreous and fundus could be detected. Under the use of duboisine the pupil was kept dilated and the pericorneal redness slowly disappeared; but otherwise no change occurred during the next ten days. In the night of November 24 a fire breaking out in the neighborhood, the patient stood for a long time at an open window to watch the fire and was thoroughly chilled. The eye became very red, painful and tender to the touch. All these symptoms, however, had subsided under warm fomentations and absolute rest in the dark room, when in the night of December 9 , the patient accidentally gave his eye a severe blow with the finger.

In the morning (December 10) his sight was so poor that he could not recognize any one near him; conjunctiva of the lower lid red and swollen; copious secretion of mucus ; much pericorneal injection; cornea streaked with interstitial opacities; lower part of iris swollen and dull, and the pupil not as fully dilated as before. Cocaine was used in addition to duboisine; and the eye improved so that December I 7 , the cornea was again clear and bright; V. good; and the fundus which could be seen very well with the ophthalmoscope, showed normal appearance. But this favorable condition did not last long; for on December 20 it was recorded "the pericorneal injection increasing; cornea streaky; dense exudate along lower border of pupil." And from that day the eye became worse every day; the cornea very cloudy; iris swollen, pupil 
smaller, and eyeball tender to the touch; and finally the tension showed an appreciable increase. The eye seemed doomed ; for an iridectomy under these circumstances promised no good; and without it the pupil would be closed by the inflammation, and the sight destroyed by the increased tension. What should be done to ward off the terrible fate-this was the question I anxiously sought to answer while sitting by the patient's bedside. I carefully scrutinized every symptom and the changes in the eye from day to day. Now duboisine bad been used all this time, and when with the increase of the inflammation the pupil showed a tendency to contract the mydriatic was applied more frequently; but I quickly perceived that the oftener it was instilled the worse the eye seemed to do; and I soon persuaded myself the unfavorable course the eye had been taking, was probably caused by the mydriatic. Its use was, therefore, stopped at once and the artificial leech was applied to the temple; and in two days the most alarming symptoms had disappeared, and the pupil instead of getting smaller remained as large as it was originally before any mydriatic was used, and has retained this size to this day.

After the duboisine was discontinued the eye never showed so serious and alarming symptoms, although all through the months of January, February and March it showed frequent attacks of slight corneal and iridic inflammation, to counteract which I relied exclusively on leeches which were applied alternately to the temple and to the neighborhood of the inner canthus, So telling was the immediate effect upon the appearance of the eye that the patient's wife, who proved an excellent nurse as well as a good observer, noticed it at once, and several times when she saw the pericorneal injection return she applied leeches with the desired success without first waiting for my order. They were applied at least a dozen times and not in one single instance did they fail. Just to give an illustration of their prompt action, I will copy the memorandum for January :

January 6, i 888 . Eye slightly redder, and watery; pain over eyebrows; but Tn. Leeches. January 7. No pain; redness almost gone.

January I 3. Pericorneal injection increasing since yesterday; cornea getting hazy and iris dull. Leeches to nose.

January I4. Injection very faint; cornea clearer; iris brighter.

January 19. Since yesterday eye red again; cornea duller. Leeches.

January 20. Less redness ; cornea brighter.

January 22. Injection again increasing.

January 23. Eye still more irritated, getting quite tender. Leeches.

January 24. The eye quite comfortable; injection greatly dimished; cornea much brighter.
In February and March the free intervals grew longer and after the beginning of April the eye remained free from irritation. By April I4 the cornea had cleared up so that an ophthalmoscopic examination (the first since November) could be obtained; it showed a normal fundus. But the test of the sight brought another surprise, inasmuch as the eye which before had a H. $\frac{1}{13}$, now showed M. $\frac{1}{3 \overline{6}} ; \mathrm{V} .=\frac{2 \pi}{4}$; and while then it required + I 2 for reading, now + I 8 was the best reading glass.

At the last examination, February 29, 1889 , the same degree of $M \cdot \frac{1}{36}$ V. $\frac{2}{4} \frac{0}{0}$ was found. This remarkable change of refraction from $H$. to $M$. might have been brought about in two ways: either the whole lens might have been pushed forward, or the convexity of its anterior surface might have been increased. The latter presumption seems to me the more plausible explanation ; for we can well imagine that the long continued inflammation of the pars ciliaris choroideæ had resulted in a permanent contraction of the ciliary fibres, by which the tension of the zonula was released and the lens allowed to become more convex. The appearance of the iris seemed to indicate that such changes had occurred; for its periphery was decidedly retracted so that the "filtration angle" was much larger than under normal circumstances, while the bulk of the iris stretched over the anterior surface of the lens like a tight-fitting glove, was so strongly convex that it formed a very sharp angle with the retracted periphery. The tension was normal; lens and vitreous were perfectly transparent and the fundus presented a normal appearance.

And here is another interesting point. If the tension of the vitreous chamber is controlled by constant flow of fluid from that cavity through the zonula into the aqueous chambers, how can we account for the fact that tension remained normal in this case, when the drainage was completely blocked by the iris being firmly glued to the anterior capsule of the lens? Is it possible that the filtration at the optic disc, which under ordinary circumstances seems to be very insignificant, may have become more active when the ordinary route was obstructed?

To complete the history of this case I have to give a brief report of the examination of the enucleated eye. It contained a large melanotic sarcoma springing from the nasal side of the fundus, and filling the larger portion of the vitreous chamber. Forward the growth had advanced to the ciliary ring; outward it was just on the point of breaking through the sclera, and backwards it reached to a line $6 \mathrm{~mm}$. from the optic papilla. The nasal half of the choroid had entirely disappeared in the tumor; the temporal portion exhibited all signs of an intense plastic cyclo-choroiditis. The retina was completely detached and the vitreous, of course, gone. Under the micros- 
cope the tumor showed the well-known structure of round-cell sarcoma with an abundance of pigment cells ; in places these prevailed to such an extent as to form deep black spots. The papilla and optic nerve were not invaded by the sarcoma and showed no structural changes; the sheath of the nerve was swollen and its connective tissue contained numerous new cells; the intervaginal space was greatly distended and filled with fibrine and lymph cells.

In conclusion I wish to say that although the sympathetic inflammation became manifest after the enucleation, it would be contrary to all our experience to assume a causative connection between this operation and the sympathetic affection. There is no case on record in which the symptoms of a sympathetic iritis were so fully developed, as in this case, five days after an injury or an operation. And I suppose if the eye had been examined between the second and fifth day the first indications of the inflammation would most likely have been noticed even at that time.

\section{THE RELATIONS BETWEEN ACNE AND DISEASES OF THE NASAL CAVITY.}

Read in the Section of Dermatology and Syphilography at the Fortieth Annual Meeting of the American Medical Association, June, 1889 . BY CARL, SEILER, M.D., OF PHILADELPHIA.

I bring before you, gentlemen, a subject which should be of a great deal of interest to you all as dermatologists, and it is of particular interest to me as a rhinologist. It has been my fortune, or perhaps misfortune, during the last ten years to observe a large number of cases of acne among the better to do class of people whose ages ranged from fourteen to fifty years, and I have noticed that the acne has borne a certain relation to the condition of the nasal chambers, becoming worse with a head-cold, gradually disappearing as the nasal irritation has disappeared, finally becoming entirely well when the nasal disease was cured, and that too without any external application or internal treatment. I made this $o b$ servation, as I have said, in a large number of cases, and about eighteen months ago $I$ made mention of the fact in a communication to the county medical society of Philadelphia. The point I would like to raise here (as of course I cannot teach you anything about acne, about its varieties, the treatment, etc.) is that acne punctata is almost always associated with atrophic rhinitis, while acne rosacea is almost always associated with hypertrophic rhinitis. You know that the nerve supply of the nose is intimately connected with the vaso-motor system, coming as it does from Meckel's ganglion and being distributed to the skin of the face, neck, etc. Again, you also know that there is a large amount of erectile tissue in the nose though opinions differ as to its function. I hold that the erectile tissue has two functions. Its function in the penis is, of course, evident, but not so with that in the clitoris, vulva, or nose. Still, it must have a function, and I think it is that of a safety-valve or overflow, to relieve local congestion or venous turgescence in the surrounding parts. For instance, through emotional excitement the penis becomes erect. The mental influence acts upon the vaso-motor system, increases the blood supply in that part, and the erectile tissues of the penis receive the overflow. In the same way the capillaries of the nose, face, and neck are easily dilated, as is observed in the blush of shame or blush of any kind. If there were no overflow, or if the overflow were interfered with, a continued congestion would result, and it would, of course, centre mostly around the glandular tissue. The distribution of the nerrous supply of these parts is very interesting, and $I$ hope to picture it in a chart.
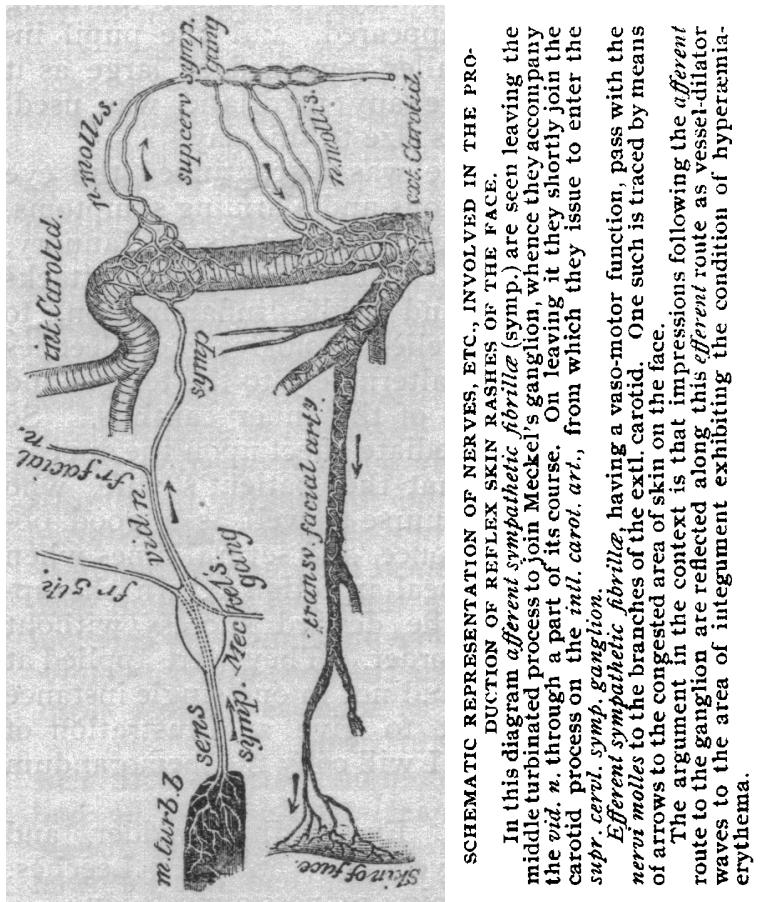

Acne usually appears about the time of puberty. At that time the involuntary erectile tissue is not yet fully developed. The influence of the nervous system is necessary for the development of the erectile tissue of the genital organs and elsewhere. In the meantime turgescence of the capillaries of the face takes place, the overflow is not taken up by the erectile tissue in the nose as this has not yet developed, and acne results. The appearance of acne in boys who practice masturbation, and its increase a day or so after the act is probably well-known to you all. I have observed it in a number of instances. 